\title{
lonising radiation metrology for the metallurgical industry
}

\author{
E. García-Toraño ${ }^{1, \star}$, F. Tzika ${ }^{2}$, O. Burda ${ }^{3}$, V. Peyrés ${ }^{1}$, M. Mejuto ${ }^{1}$, T. Crespo $^{1}$, U. Wätjen ${ }^{2}$, D. Arnold $^{3}$, V. Sochor $^{4}$, \\ A. $\mathrm{Svec}^{5}, \mathrm{P} . \mathrm{Carconi}^{6}, \mathrm{P}$. de Felice ${ }^{6}$, and J. Tecl ${ }^{7}$ \\ 1 CIEMAT - Laboratorio de Metrología de Radiaciones Ionizantes, Avda. Complutense 40, 28040 Madrid, Spain \\ 2 EC-JRC-IRMM - European Commission, Joint Research Centre, Institute for Reference Materials and Measurements, \\ 2440 Geel, Belgium \\ 3 PTB - Physikalisch-Technische Bundesanstalt, Bundesallee 100, 38116 Braunschweig, Germany \\ ${ }^{4} \mathrm{CM}$ - Cesky Metrologicky Institut, Brno, Czech Republic \\ 5 SMU - Slovenský Metrologický Ústav, Bratislava, Slovakia \\ ${ }^{6}$ ENEA - Istituto Nazionale di Metrologia delle Radiazioni Ionizzanti, Rome, Italy \\ 7 ENVINET - shareholding company, Trebic, Czech Republic
}

Received: 25 February 2014 / Accepted: 24 April 2014

\begin{abstract}
Every year millions tons of steel are produced worldwide from recycled scrap loads. Although the detection systems in the steelworks prevent most orphan radioactive sources from entering the furnace, there is still the possibility of accidentally melting a radioactive source. The MetroMetal project, carried out in the frame of the European Metrology Research Programme (EMRP), addresses this problem by studying the existing measurement systems, developing sets of reference sources in various matrices (cast steel, slag, fume dust) and proposing new detection instruments. This paper presents the key lines of the project and describes the preparation of radioactive sources as well as the intercomparison exercises used to test the calibration and correction methods proposed within the project.
\end{abstract}

Keywords: Ionising radiation measurements; interlaboratory comparisons; EURAMET; EMRP

\section{Introduction}

In 2012, about 1.4 billion tons of steel were produced worldwide of which 170 millions in the European Union (EU) [1]. A large part of it, $43 \%$ in the EU, is produced by recycling scrap. Recycling is a key factor in sustainable development and steel industry has a long tradition in this.

Radioactive material can enter into scrap by several pathways, from which sources of artificial origin are the greater concern. Industrial and medical applications of radioactivity, among others, require the use of sealed sources that, in some cases, can run out of regulatory control ("orphan sources"). Very often, such sources are contained in heavy shieldings.

Scrap loads are tested for radioactive contents before entering into the melting process using radiation portal monitors and other equipment to survey the loads. Under normal circumstances, any contaminated piece of scrap is detected at this stage. However, in some cases, because of self-shielding of the sources or radiation attenuation by the container load, radioactive sources can pass the screening procedure without being detected; in these cases, the source enters the furnace and is smelt. This results in contamination of furnace, cast steel, slag, fume dust, filters

^ Correspondence: e.garciatorano@ciemat.es and ducts, causes leaking of radioactive substances into the environment and leads to expensive clean-up costs [2].

The occurrence of incidents of this kind has been significantly reduced in the last years, but these risks still exist and, in addition to scrap controls, "a posteriori" controls are needed to guarantee and certify the absence of radioactive contamination in steel and by-products. It is worth mentioning that some products, as slag, are also recycled at large scale in construction works [3].

The MetroMetal project [4], carried out in the frame of the European Metrology Research Programme (EMRP) [5], addresses this problem by studying the existing measurement systems, developing sets of reference sources in various matrices (cast steel, slag, fume dust) and proposing new detection instruments. The project is managed by a consortium of 13 European National Metrology and Designated Institutes, one international organisation and an associated engineering company.

The project has the following scientific and technical objectives:

- The development of reliable and SI traceable methods optimised for the control/measurement of radioactivity at each stage of the smelting process (e.g. scrap loads, metal products, slag and fumes dust).

- The development of reference standards for composite steel, cast steel, slag and fume dust containing a known 
activity of some radionuclides $\left({ }^{60} \mathrm{Co},{ }^{137} \mathrm{Cs},{ }^{192} \mathrm{Ir}\right.$, ${ }^{226} \mathrm{Ra}$ and ${ }^{241} \mathrm{Am}$ ) considered as potential contaminants. They will be prepared in geometries/matrices that match the industrial environments for which they are designed.

- The design of an optimised spectrometric device for the measurement of activity in cast steel, fume dust and slag samples and the production and testing at the partner laboratories of two prototypes.

- The evaluation of both prototypes at end-user facilities (foundries).

- The development of technical recommendations for European and National Standards Committees for the standardisation of radioactivity monitoring (e.g. calibration of measurement systems, on-line monitoring of production and certification of cast steel).

This paper focuses on the preparation of radioactive sources as well as the interlaboratory comparisons (ILCs) used to test the calibration and correction methods proposed within the project. Results from some of these ILCs will be used in the final characterization of the respective radioactivity standards.

\section{Preparation of sources}

A crucial part of the project is the development of standards of contaminated materials to be used in the calibration of the surveying instruments. These sources must be:

1. Prepared in matrices similar to those found at end-user facilities and with radionuclide contents that match what is described in the literature $[6,7]$ for those matrices.

2. Compatible with actual and future measurement systems.

3. Characterized in terms of activity and/or activity concentration of the contained nuclides.

4. Traceable to national standards of activity.

To this aim, sets of sources on several matrices have been prepared by the project partners. They include composite steel, cast steel, slag and fume dust standards containing several radionuclides. The source production was followed by their characterization in terms of activity or activity concentration by independent measurements in partners' laboratories and, in certain cases, by using the results of ILCs.

Radioactivity monitoring in cast steel is carried out at steelworks on a routine basis using probes taken for other purposes such as steel composition determination (Fig. 1). There is no standard size, but probes are typically cylindrical shaped with diameters in the order of several $\mathrm{cm}$. Probes are commonly measured with scintillator-based detectors that often have a cylindrical well were the sample is placed for measurement. Slag and fume dust are checked by their radioactive contents in a variety of manners from which the most common method is to survey the slag and fume dust loads with large detection portals when they are removed from steelworks premises.

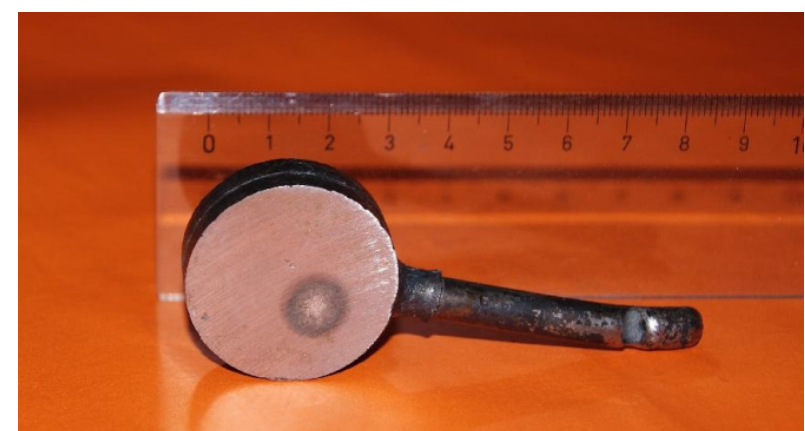

Fig. 1. Typical cast-steel probe used at steelworks for analysis and quality control.

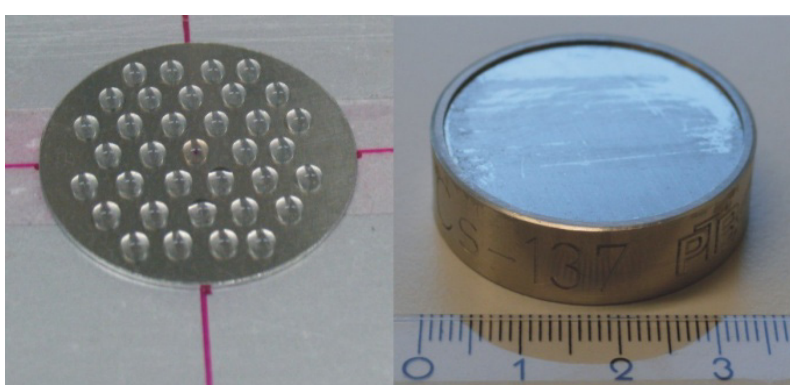

Fig. 2. Composite steel source with ${ }^{137}$ Cs. Left: steel plate with 35 drops of a ${ }^{137} \mathrm{Cs}$ solution put in a regular pattern with a spacing of about $5 \mathrm{~mm}$. Right: ready calibration source with engraved outer case.

Since one of the pre-requisites for source design was the compatibility with existing measuring systems, it was decided that reference sources would have cylindrical shapes and their diameter should be less than $10 \mathrm{~cm}$. With these dimensions, sources would adapt well to most measuring systems actually in the market.

Composite cast steel reference standards with ${ }^{241} \mathrm{Am},{ }^{137} \mathrm{Cs}$ and ${ }^{60} \mathrm{Co}$ activity concentrations of about $1 \mathrm{~Bq} / \mathrm{g}$ and $10 \mathrm{~Bq} / \mathrm{g}$ were produced. Source consists of a stack of 11 thin steel plates (diameter $34 \mathrm{~mm} \times$ height $0.8 \mathrm{~mm}$ ) inserted in a supporting outer case (diameter $35 \mathrm{~mm} \times$ height $15 \mathrm{~mm}$ ). Ten of eleven plates were covered from one side with a double-sided adhesive polyethylene foil. For the preparation of these sources, 35 drops of similar mass were taken with a pipette from a calibrated solution and put onto each of ten plates in a regular pattern with a spacing of about $5 \mathrm{~mm}$ (Fig. 2, left).

After drying, the plates were placed together in the supporting outer case (Fig. 2, right). The main advantage of a composite reference standard is that the activity on the steel disks is determined in a traceable way with a relative uncertainty of less than $1 \%$ by using drops of a standardized activity solution.

Real cast steel samples contaminated with ${ }^{60} \mathrm{Co}$ were provided partially by the Siempelkamp Nukleartechnik GmbH, Krefeld, Germany [8] and VUHZ, Czech Republic [9]. Siempelkamp company melted mixed contaminated metal scrap of about $1 \mathrm{t}$, selected one batch 


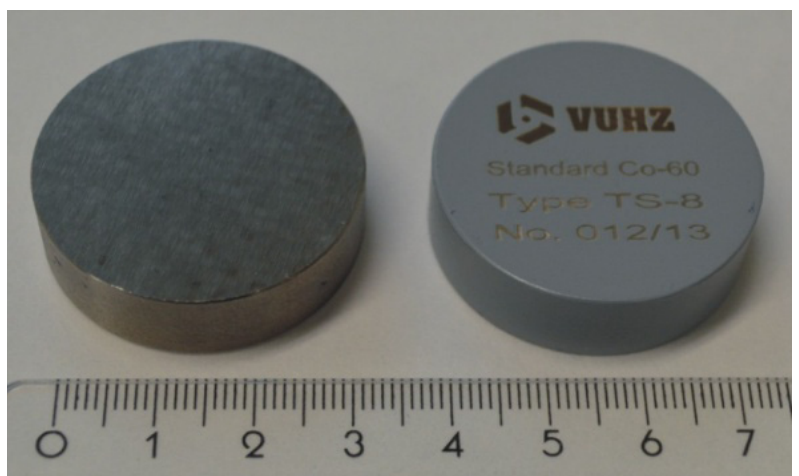

Fig. 3. Cast steel sources containing ${ }^{60}$ Co. Left: Siempelkamp. Right: VUHZ.

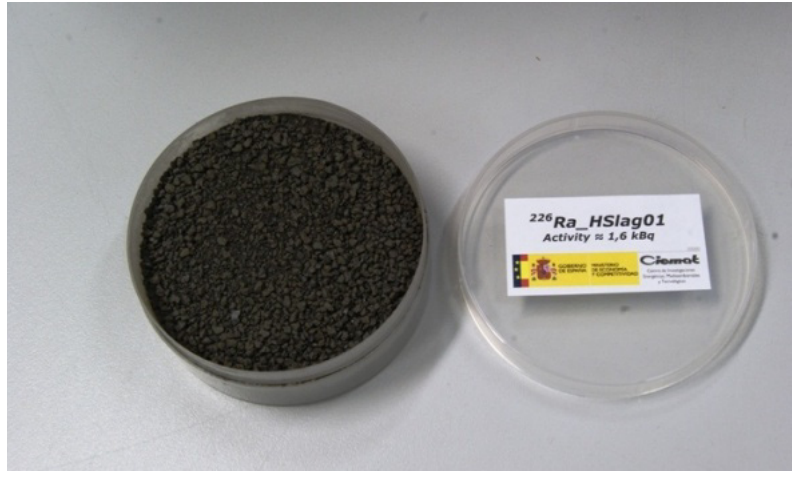

Fig. 4. Slag source spiked with ${ }^{226} \mathrm{Ra}$ in the container.

with a Co-60 activity concentration of $1 \mathrm{~Bq} / \mathrm{g}$, cast one rod and cut into disks (Fig. 3, left). Due to a high turbulence in an electric induction furnace, the contaminant radionuclide is homogeneously distributed in the molten metal. The chemical composition of these samples was determined by project partners.

The VUHZ samples (Fig. 3, right) were prepared from the S355 steel according to EN 10025 by melting in an electric induction furnace and contaminated adding ${ }^{60} \mathrm{Co}$ in some metallic alloy.

Then casted cylindrical pieces were turned and cut into proper dimensions. Both types of samples have a diameter of $35 \mathrm{~mm}$ and a height of $10 \mathrm{~mm}$.

At CIEMAT, two sets of slag sources were prepared by spiking slags with a solution of ${ }^{226}$ Ra. Raw material was collected from a electric-arc furnace steel foundry near Madrid and after characterization was passed over a $2 \mathrm{~mm}$ screen, the standard size for reference soils [10,11]. Before spiking, its radioactive content was determined by gammaray spectrometry [12]. Slag was bottled in 15 polypropylene cylindrical containers $(r=3.75 \mathrm{~cm}, h=3 \mathrm{~cm})$ (Fig. 4). Each source contained approximately $160 \mathrm{~g}$ of material. Spiking was carried out with a ${ }^{226}$ Ra standard solution following an immersion procedure similar to that described by Shakhashiro et al. [13]. Full details of the preparation, spiking and homogeneity testing by gammaray spectrometry are described elsewhere [14]. Figure 3 presents a typical source. Two sets were prepared with nominal activity concentrations of 1 and $10 \mathrm{~Bq} / \mathrm{g}$.

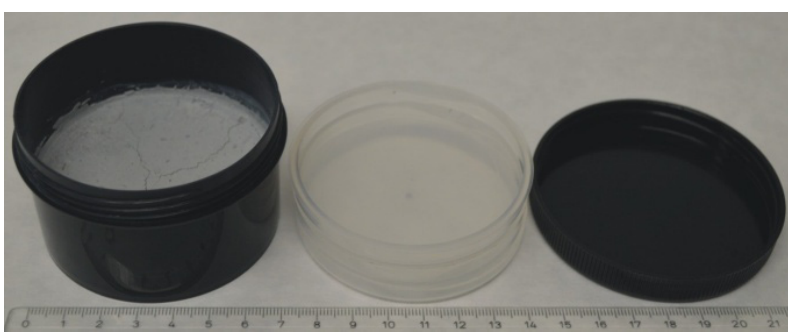

Fig. 5. Fume dust source contaminated with ${ }^{137} \mathrm{Cs}$ in the container (left) is hermetically closed with covers (middle and right).

Fume dust material contaminated with ${ }^{137} \mathrm{Cs}$ was provided by Siempelkamp. This powder-like homogenous material (Fig. 5) was put into a polyethylene container (diameter $6.95 \mathrm{~cm}$, height $1.87 \mathrm{~cm}$, wall thickness $1 \mathrm{~mm}$ ). In order to ensure better experimental reproducibility, the fume dust (50.2 g) was compressed by a factor of 1.84 (using 3 transparent covers, Fig. 5, middle) and hermetically closed (with black cover, Fig. 5, right), so that the sample filling height is well fixed. Thus, the correction factors for self-attenuation, coincidence summing, efficiency and geometry transfer can be defined better than $2 \%$.

\section{Inter-laboratory comparisons}

Four interlaboratory comparisons (ILCs) were conducted aiming to test the calibration and correction methods proposed within the project. The ILCs involved the determination of

(a) ${ }^{60} \mathrm{Co}$ in two types of cast steel, of different origin, of lower activity $(\sim 1 \mathrm{~Bq} / \mathrm{g})$;

(b) ${ }^{226} \mathrm{Ra}$ in slag of higher activity $(\sim 10 \mathrm{~Bq} / \mathrm{g})$; and

(c) ${ }^{137} \mathrm{Cs}$ in fume dust of higher activity $(\sim 10 \mathrm{~Bq} / \mathrm{g})$.

The ILC for the cast steel involved both sets (produced at Siempelkamp and at VUHZ) of low activity cast steel samples developed.

The ILCs were coordinated by EC-JRC-IRMM and all 14 project partners participated in them except the partners responsible for the production of standards. The overall organization and related time schedule of all four ILCs were agreed among partners beforehand in the form of a flowchart. Each ILC was implemented in a similar structure:

- Preparation of adequate number of samples and homogeneity testing at the partners laboratories.

- Distribution of samples, technical protocols and reporting templates to participants.

- Measurements of samples at partners' laboratories.

- Reporting of the activity concentration results to JRC.

- Final comparison and evaluation of reported results.

The inhomogeneity in the radionuclide concentration in a comparison material increases the uncertainty of the corresponding reference value. The standard uncertainty contribution from the homogeneity study, $u_{h o m}$, will be 
Table 1. Standard uncertainty contributions due to inhomogeneity, $u_{\text {hom }}$ (in \%), for the different ILC materials.

\begin{tabular}{cccc}
\hline ILC material & $N$ & $n$ & $u_{\text {hom }}$ \\
\hline Cast steel Siempelkamp & 20 & 1 & 1.08 \\
Cast steel VUHZ & 14 & 2 & 0.35 \\
Slag & 14 & 4 & 1.1 \\
Fume dust & 13 & 1 & 0.35 \\
\hline
\end{tabular}

taken into account, along with the one from the activity characterization, $u_{c h a r}$, in the estimation of the combined standard uncertainty of the reference activity value of each material, $u_{r e f}$, by using the following formula:

$$
u_{\text {ref }}=\sqrt{u_{\text {char }}^{2}+u_{\text {hom }}^{2}} .
$$

It must be noted that uncertainty due to instability is disregarded because the types of the materials are considered to not suffer changes with time or transport. The homogeneity study was performed in one partner laboratory for each candidate standard. The adopted $u_{\text {hom }}$ components for the ILC materials are given in Table 1 along with the corresponding number of samples, $N$, and the number of measurements per sample, $n$, performed for each material. In this table the values for $u_{h o m}$ correspond to the standard deviation between $n \times N$ measurements.

For each ILC, JRC distributed one sample per participant and the technical protocol including sample information, instructions and relevant dates. The elemental composition of the raw materials was provided as a common basis for the participants conducting Monte Carlo simulations for relative or absolute efficiency calibration of their gamma-ray spectrometry systems. The participants were recommended to analyze the samples by HPGe based gamma-ray spectrometry. Different efficiency calibration methods (experimental, Monte Carlo and combined ones) were used.

During the reporting, participants were requested to provide detailed information on their measurement systems and procedures as well as references to their data sources to facilitate evaluation of the ILCs results and ensure traceability of activity determination. Up to date, they have reported their results for the two ILCs for ${ }^{60} \mathrm{Co}$ in Siempelkamp and VUHZ cast steel which are shown in Figures $6 \mathrm{a}$ and $6 \mathrm{~b}$, respectively.

The results were statistically treated to derive the power-moderated mean for activity concentration following the official CCRI(II) (Consultative Committee for Ionising Radiation) procedure for key comparisons [15].

The mean was calculated to be $(1.077 \pm 0.015) \mathrm{Bq} / \mathrm{g}$ and $(1.483 \pm 0.022) \mathrm{Bq} / \mathrm{g}$, for Siempelkamp and VUHZ cast steel, respectively, where the stated uncertainties correspond to the standard deviation of the power-moderated mean.

In each comparison one result was excluded from the calculation of the mean on the basis of reported, prior to the disclosure of the results, technical problems with the detector system resulting in count rate loses. Ten out of 13 and 11 out of 13 results of ILC on Siempelkamp and

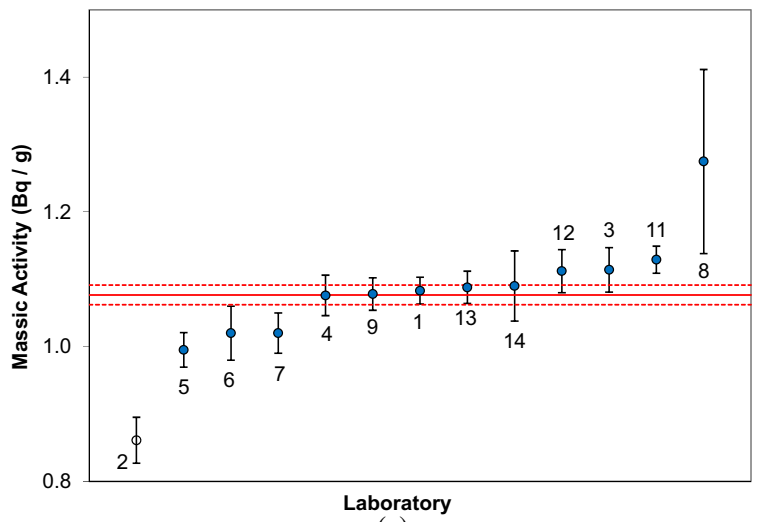

(a)

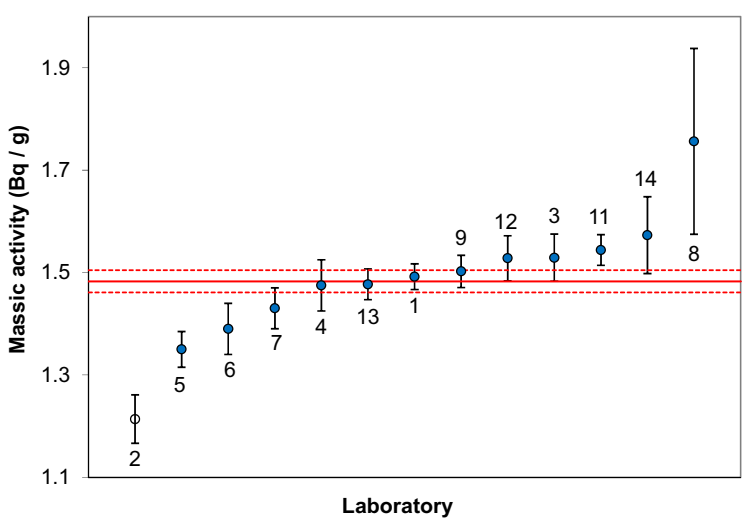

(b)

Fig. 6. Laboratory results of ${ }^{60} \mathrm{Co}$ activity concentration in (a) Siemplekamp and (b) VUHZ cast steel, with combined standard uncertainties, sorted in ascending order. The solid line represents the power-moderated mean and the dotted lines the $\pm 1 \sigma$. The hollow data point was excluded from the calculation of the mean.

VUHZ cast steel samples, respectively, agreed with the mean values within the stated experimental uncertainties $(k=1)$.

One partner has reported higher uncertainty values in both ILCs. These uncertainties are realistic and, according to the reported uncertainty budgets, their main contribution comes from reproducibility. The overall comparison between the results of the two ILCs revealed a similar pattern due to the fact that the same radionuclide was determined in samples of similar dimensions and matrix.

The individual laboratory performance was expressed in terms of relative deviations and $E_{n}$ numbers [16].

Results from some of these ILCs will be used in the final characterization of the respective radioactivity standards.

\section{Conclusions}

Results presented in the precedent section indicate that the first steps of the project have been progressing successfully. As indicated in the introduction, the development and characterization of the sources is a necessary stage for the development of subsequent phases of the project. 
Significant progress was also made in the design and implementation of a new instrument of improved performance. As a result two prototypes, based on semiconductor detectors, are currently being assembled at partner's laboratories. This equipment will be calibrated for the most common operational conditions using a set of sources, the production of some of which has been described in this paper. Monte Carlo calculations will be carried out to obtain additional calibration factors covering a wider range of experimental conditions. The final stage of the project will be the operation and demonstration of both prototypes at end-user facilities in Europe.

Acknowledgements. This work is supported by the European Union under the Joint Research Project IND04 MetroMetaL within the European Metrology Research Programme (EMRP). The EMRP is jointly funded by the EMRP participating countries within EURAMET and the European Union. We would like to thank the Siempelkamp Nukleartechnik Krefeld for providing us with real contaminated materials.

\section{References}

1. World Steel Association, http://www . worldsteel.org

2. D.S. Harvey, K. Baldry, A. Bishop, Radiation safety following accidental melting of radioactive sources at steelplants. Rev. Metall. 101, 285-289 (2004)

3. B. Das, S. Prakash, P.S.R. Reddy, V.N. Misra, An overview of utilization of slag and sludge from steel industries, Resour. Conserv. Recycl. 50, 40-57 (2007)

4. Ionising radiation metrology for the metallurgical industry, http://projects.ciemat.es/web/metrometal

5. EMRP, European Research Metrology Programme, http://www.emrponline.eu/
6. UNECE, EC, IAEA, Report on the Improvement of the Management of Radiation Protection Aspects in the Recycling of Metal Scrap. ECE/TRADE/278, 2001

7. K. Baldry, D.S. Harvey, A. Bishop, Accidental Melting of Radioactive Sources, Proceedings of IRPA 11 (Madrid, 2011)

8. http://www . siempelkamp.com/index .php?id=2003\&L=0

9. http://www.vuhz.cz/

10. ISO, Certification of reference materials, General and statistical principles, Guide 35. Int. Organ. Stand., Geneva, Switzerland (2006)

11. ISO, General requirements for the competence of reference material producer - Guide 34. Int. Organ. Stand., Geneva, Switzerland (2000)

12. M. Sahagia, A. Luca, R.M. Mãrgineanu, N. Navarro, V. Peyrés, B. Pérez López, E. Garcia-Toraño, J.A. SuárezNavarro, Determination of the content of natural radionuclides in furnace slag used for the preparation of standard sources. J. Radioanal. Nucl. Chem. 298, 2037-2042 (2013)

13. A. Shakhashiro, A.M. Gondin da Fonseca Azeredo, U. Sansone, A. Fajgelj, Matrix materials for proficiency testing: optimization of a procedure for spiking soil with gamma-emitting nuclides. Anal. Bioanal. Chem. 387, 2509-2515 (2007)

14. M. Mejuto, M.T. Crespo, E. García-Toraño, V. Peyrés, M. Roteta, L. Pérez del Villar, Preparation and characterization of a ${ }^{226} \mathrm{Ra}$ spiked slag as reference material for radioactive control of steelworks, Appl. Radiat. Isot. 94, 166-174 (2014)

15. S. Pommé, Determination of a reference value, associated standard uncertainty and degrees of equivalence, JRC Scientific and Policy report EUR 25355 EN, 2012, ISBN 978-92-79-25104-7

16. ISO, ISO/IEC 13528, Statistical methods for use in proficiency testing by interlaboratory comparisons. Int. Organ. Stand., Geneva, Switzerland (2005) 